\title{
Lysates of Metschnikowia Yeast with Higher Content of Hydroxyproline
}

\author{
Ewelina Pawlikowska, Monika Szymańska, Joanna Berłowska, and Dorota Kręgiel* \\ The chemical characteristics of lysates obtained from yeasts belonging to \\ Metschnikowia spp. were determined. Cell lysis was induced using \\ saponin from $Q$. saponaria or $5 \% \mathrm{NaCl}$. The process was conducted \\ at $50{ }^{\circ} \mathrm{C}$ for $48 \mathrm{~h}$. The enzymatic profiles of the resulting lysates were \\ analyzed. The mannose and glucose contents were also investigated, as \\ well as the concentrations of proteins, free amino nitrogen (FAN), and \\ free amino acids. The results were compared to the characteristics of \\ lysates from conventional industrial strains of Saccharomyces spp. \\ obtained under analogous conditions. The Metschnikowia lysates \\ showed different chemical profiles and the pool of individual amino \\ acids was generally smaller. However, the content of hydroxyproline \\ HPro was 4 to 5 times higher. The results of this study show that yeast \\ lysates are an attractive supplement for numerous applications.
}

Keywords: Metschnikowia; Autolysates; Amino-acid profile; Hydroxyproline

Contact information: Department of Environmental Biotechnology, Lodz University of Technology, 90-924 Lodz, Poland; *Corresponding author: dorota.kregiel@p.lodz.pl

\section{INTRODUCTION}

Yeast lysates are used in the medical, food, and cosmetics industries. They are a source of carbohydrates, proteins, and free amino acids, which may be used as substrates for the biosynthesis of various compounds, such as flavors, hormones, coenzymes, or neurotransmitters. Yeast lysates are obtained mainly from yeast of the genus Saccharomyces. Yeast propagated for this purpose, or post-fermentation yeast, left over from the production of beer or wine, may be used. It is then submitted to slow natural autolysis or a much faster induced process. Induced lysis is deliberately caused by the effects of temperature, high pressure homogenization, ultrasound treatment, or chemical inducers (enzymes, detergents, salts, or alcohols). Rarely, other industrial yeasts from the genera Kluyveromyces, (Lukondeh et al. 2003; Berłowska et al. 2015), Scheffersomyces/ Pichia (Bartolo-Aguilar et al. 2017; Berłowska et al. 2017), or Yarrowia (Pozo-Dengra et al. 2006) may be utilized. The chemical composition of the obtained lysates depends on the method of cell disruption as well as on the genera or species of the yeast (Berłowska et al. 2015; Berłowska et al. 2017; Comuzzo et al. 2017; Bertolo et al. 2019; Jacob et al. 2019).

The yeast genus Metschnikowia is included on the list of biotechnologically useful microorganisms prepared by scientists and the food industry (Bourdichon et al. 2012). This non-conventional yeast is recommended for the release of volatile thiols and terpenes in white wines, increasing their aromatic intensity (Mas et al. 2016). For example, an increase in the levels of the thiol 4-MSP (4-methyl-4-sulfanylpentan-2-one) and a decrease in higher alcohol content, may give important impact on white wines, making them fruitier and fresher (Ruiz et al. 2018). Strains of Metschnikowia have been used in the production of 
low alcohol wines (Varela et al. 2017), and their potential for the deoxidation of fermentation wort has also been recognized (Pawlikowska et al. 2019b). Metschnikowia strains have been identified as a possible biocontrol agent against Botrytis and numerous post-harvest fruit diseases (Sipiczki 2006; Pawlikowska et al. 2019a). However, despite the growing interest in Metschnikowia strains, there are no data on the characteristics of their autolysates. Therefore, this research studied the enzymatic profiles, proteins, and amino acid profiles of autolysates produced from Metschnikowia strains. Autolysis was chemically assisted in both conventional and non-conventional ways, using $\mathrm{NaCl}$ and saponins from Quillaja saponaria (Berłowska et al. 2015; Berłowska et al. 2017). The researchers compared the results for Metschnikowia spp. with the characteristics of yeast autolysates from classical strains of Saccharomyces spp. This is the first report on the chemical characteristics of Metschnikowia spp. lysates.

\section{EXPERIMENTAL}

\section{Yeast Strains and Cultivation}

Biomass from three yeast strains belonging to Metschnikowia spp were used: the collection strain $M$. pulcherima NCYC2321 (Norwich, UK) and two isolates, $M$. andauensis D2 (GenBank MK612095), and M. sinensis D9 (GenBank MK612102) (Pawlikowska et al. 2019a). Two strains of Saccharomyces spp. were also utilized: the winery strain $S$. cerevisiae Tokay, and the brewery yeast $S$. cerevisiae TT from LOCK105 Culture Collection (Lodz, Poland). The yeasts were cultured in $50 \mathrm{~mL}$ of wort broth (Merck) in $500 \mathrm{~mL}$ round bottom flasks at $25{ }^{\circ} \mathrm{C}$ for $72 \mathrm{~h}$ on a rotary shaker at $220 \mathrm{rpm}$. After cultivation, the cells were washed twice with sterile distilled water by centrifugation $\left(10{ }^{\circ} \mathrm{C}, 10 \mathrm{~min}, 3000 \times \mathrm{g}\right.$, Eppendorf 5804R, Hamburg, Germany). The concentration of yeast cells was determined via counting with a hemocytometer and standardized to $10^{9}$ cells/mL.

\section{Induced Lysis}

The yeast biomass was suspended in distilled water with a ratio of 1:1. Saponins from $Q$. saponaria (Sigma Aldrich, St. Louis, MO, USA) were then added to a final concentration of $0.08 \% \mathrm{w} / \mathrm{v}$, as an inducer of cell lysis. The cell lysis was conducted in 500 $\mathrm{mL}$ Erlenmeyer flasks on a laboratory shaker $(112 \mathrm{rpm})$ at $52 \pm 2{ }^{\circ} \mathrm{C}$ for $48 \mathrm{~h}$ (Heidolph Unimax 1010, Schwabach, Germany). Then, the solid residue was centrifuged $\left(5^{\circ} \mathrm{C}, 15\right.$ min, 3500×g; Eppendorf 5804R, Hamburg, Germany) and the supernatant was collected.

\section{Protein Concentration}

The protein content in the yeast lysates was analyzed using a Direct Detect ${ }^{\circledR}$ System (Merck-Millipore, Waltham, MA, USA). This method is based on Fourier transform infrared spectroscopy (FTIR), which detects amide bonds in polypeptide chains (Berlowska et al. 2017).

\section{FAN Determination}

The free amino nitrogen concentration (FAN) was determined based on the color reaction of amino acids with ninhydrin and by absorbance measurements at a wavelength of $570 \mathrm{~nm}$. The concentration of ammonia nitrogen was measured using the colorimetric method with Nessler's reagent at a wavelength of 400/425 nm (Berlowska et al. 2015). 


\section{Free Amino Acid Profiles}

To remove the protein fraction with a molecular weight over $3 \mathrm{kDa}$, the yeast lysate was centrifuged at $25^{\circ} \mathrm{C}$, speed 10,000 g, 2 h; 5804R (Eppendorf, Hamburg, Germany) and filtered using Amicon ${ }^{\circledR}$ Ultra-4 Ultracel-3 membranes (Merck-Millipore, Massachusetts, USA). The samples were then derivatized using phenylisothiocyanate to form phenylthiocarbamyl amino acids (Waters Workstation, Massachusetts, USA). The derivatived forms were analyzed by HPLC (diluent WAT088119, Eluent A WAT052890, Eluent B WAT088112, Pico Column $3.9 \times 300 \mathrm{~mm}$, time $20 \mathrm{~min}$; Waters, Waltham, MA, USA, Thermo Finnigan Surveyor HPLC System, Waltham, MA, USA). The amino acids content were expressed in pmol units (Berlowska et al. 2017).

\section{Enzymatic Profiles}

The enzymatic profiles of the yeast suspensions, including 19 different enzymes, were estimated after $24 \mathrm{~h}$ of autolysis using the API ZYM test (BioMerieux, Lyon, France). Inoculation and evaluation were carried out based on the manufacturer's instructions and recommendations. Only those suspensions that demonstrated visible changes in the color of the medium were considered to show enzymatic activity. The intensity of the color reflected the amount of degraded substrate. Values ranged from ' 0 ', no activity, to ' 3 ', maximum activity (Pawlikowska et al. 2019a).

\section{Mannose and Glucose Content}

The monosaccharide profiles of the obtained yeast lysates were analyzed using a UV-spectrophotometer (Thermo Scientific Multiskan GO; Thermo Fisher Scientific, Munich, Germany) and a Megazyme K-MANGL Kit for glucose and mannose determination. The assays were conducted according to the manufacturer's instructions (Berlowska et al. 2017).

\section{Statistics}

Means were calculated from the data obtained from three independent experiments and the standard deviation (SD) was calculated.

\section{RESULTS AND DISCUSSION}

In this study, researchers used both classical yeasts belonging to Saccharomyces spp. and non-conventional yeasts from Metschnikowia spp. Solutions of $\mathrm{NaCl}$ and plant saponin from $Q$. saponaria were used as lysis inducers. In previous studies on yeast lysis conducted by Berlowska et al. (2017), it was observed that saponins from $Q$. saponaria promote the autolysis of different yeast genera: Saccharomyces, Kluyveromyces, Scheffersomyces, and Pichia. This analysis studied the activities of the enzymes in yeast suspensions undergoing induced lysis. Table 1 presents the results of enzymatic fingerprinting obtained after $24 \mathrm{~h}$ of incubation, in the middle of the lysis process.

The use of API Zym enabled assessment of the activity of the enzymes responsible for catalyzing protein hydrolysis (trypsin, $\alpha$-chymotrypsin, leucine arylamidase, valine arylamidase, and cystine arylamidase), ester bond hydrolysis (esterase C4, esterase, and lipase), dephosphorylation reactions (alkaline phosphatase, acid phosphatase, and naphthyl-AS-BI-phosphohydrolase). 
Table 1. Enzymatic Activity of Yeast Cell Suspensions Determined by the Api Zym Assay

\begin{tabular}{|c|c|c|c|c|c|c|c|c|c|c|c|}
\hline \multicolumn{2}{|c|}{ Enzyme } & \multicolumn{10}{|c|}{ Yeast strain } \\
\hline \multirow{2}{*}{ Classes } & \multirow{2}{*}{$\begin{array}{c}\text { Specific } \\
\text { activity }\end{array}$} & \multicolumn{2}{|c|}{ NCYC } & \multicolumn{2}{|c|}{ D2 } & \multicolumn{2}{|c|}{ D9 } & \multicolumn{2}{|c|}{ Tokay } & \multicolumn{2}{|c|}{ TT } \\
\hline & & $\mathrm{NaCl}$ & $\mathbf{S}$ & $\mathrm{NaCl}$ & $\mathbf{S}$ & $\mathrm{NaCl}$ & $\mathbf{S}$ & $\mathrm{NaCl}$ & $\mathbf{S}$ & $\mathrm{NaCl}$ & $\mathbf{S}$ \\
\hline \multirow{4}{*}{ Proteases } & Leucine arylamidase & 0 & 0 & 1 & 1 & 1 & 0 & 0 & 0 & 0 & 0 \\
\hline & Valine arylamidase & 0 & 0 & 1 & 1 & 0 & 0 & 3 & 3 & 3 & 3 \\
\hline & Cystine arylamidase & 2 & 1 & 0 & 0 & 0 & 0 & 1 & 1 & 1 & 1 \\
\hline & Trypsin & 0 & 0 & 0 & 0 & 0 & 0 & 1 & 0 & 0 & 0 \\
\hline \multirow{2}{*}{ Lipases } & Esterase (C4) & 0 & 0 & 0 & 0 & 0 & 0 & 1 & 1 & 3 & 3 \\
\hline & Esterase lipase & 0 & 0 & 0 & 0 & 0 & 0 & 1 & 1 & 1 & 1 \\
\hline \multirow{3}{*}{ Phosphatases } & Alkaline phosphatase & 2 & 1 & 1 & 1 & 1 & 2 & 0 & 0 & 0 & 0 \\
\hline & Acid phosphatase & 3 & 3 & 3 & 3 & 3 & 3 & 0 & 0 & 1 & 1 \\
\hline & $\begin{array}{c}\text { Naphtol-AS-BI- } \\
\text { phosphohydrolase }\end{array}$ & 2 & 1 & 1 & 2 & 1 & 1 & 3 & 3 & 3 & 3 \\
\hline \multirow{4}{*}{ Glycosidases } & a-Galactosidase & 0 & 0 & 0 & 0 & 0 & 0 & 2 & 2 & 1 & 1 \\
\hline & $\beta$-Glucuronidase & 0 & 0 & 0 & 0 & 0 & 0 & 0 & 0 & 0 & 2 \\
\hline & a-Glucosidase & 0 & 0 & 0 & 0 & 0 & 0 & 2 & 3 & 0 & 0 \\
\hline & $\alpha$-Mannosidase & 0 & 0 & 0 & 0 & 0 & 1 & 0 & 0 & 0 & 2 \\
\hline
\end{tabular}


The assay also evaluated binding of carbohydrate ( $\alpha$-galactosidase, $\beta$-galactosidase, $\beta$-glucuronidase, $\alpha$-glucosidase, $\beta$-glucosidase, $\mathrm{N}$-acetyl- $\beta$-glucosaminidase, $\alpha$-mannosidase, and $\alpha$-fucosidase). During autolysis, researchers noted the highest activity of phosphatases in the Metschnikowia spp. suspensions. Recent insights into the function and regulation of these enzymes have unveiled extremely interesting aspects of yeast biology. For example, phosphatases regulate salt tolerance, G1/S cell cycle transition, and cell integrity, as well as being involved in protein synthesis (Arino 2002). Their activity, which was significant in the middle of the autolysis process, indicates the occurrence of many cell defense processes under strong environmental stress (temperature, osmotic pressure, and cell density). In the autolyzed mixtures, the activity of arylamidases was observed, which proves the occurrence of protein hydrolysis. In the yeast mixtures, no glycosidase activity was detected.

The enzymatic profiles of the conventional yeast strains varied. In Saccharomyces spp. suspensions researchers noted higher activity for some proteases (valine arylamidase and cystine arylamidase), lipases (esterase C4 and esterase), and some carbohydratebinding enzymes ( $\alpha$-galactosidase, $\beta$-glucuronidase, $\alpha$-glucosidase, and $\alpha$-mannosidase). Very high activity was observed for one phosphatase using naphthol-AS-BI-phosphate. These activities indicate the progressive degradation of proteins, lipids, and carbohydrates, namely glucanes and mannanes - the main constituents of yeast cell wall (Berlowska et al. 2016).

After the final process of autolysis, the contents of proteins, free amino nitrogen (FAN), and monosaccharides (glucose and mannose) in the yeast lysates were measured. The results are presented in Table 2 .

Table 2. General Characteristics of Yeast Lysates

\begin{tabular}{|c|c|c|c|}
\hline Yeast strain & Lysis inducer & $\begin{array}{c}\text { Protein } \\
(\mathbf{m g} / \mathbf{m L})\end{array}$ & $\begin{array}{c}\text { FAN } \\
\mathbf{( m g / m L})\end{array}$ \\
\hline \multirow{2}{*}{ M. pulcherrima NCYC2321 } & $\mathrm{NaCl}$ & $4.48 \pm 0.56$ & $1131.34 \pm 23.45$ \\
\cline { 2 - 4 } & saponin & $3.51 \pm 0.26$ & $831.34 \pm 67.34$ \\
\hline \multirow{2}{*}{ M. andauensis D2 } & $\mathrm{NaCl}$ & $5.48 \pm 0.24$ & $1127.34 \pm 45.67$ \\
\cline { 2 - 4 } & saponin & $2.53 \pm 0.09$ & $1029.34 \pm 67.65$ \\
\hline \multirow{2}{*}{ M. sinensis D9 } & $\mathrm{NaCl}$ & $4.76 \pm 0.35$ & $656.66 \pm 45.34$ \\
\cline { 2 - 4 } & saponin & $3.16 \pm 0.12$ & $666.66 \pm 56.78$ \\
\hline \multirow{2}{*}{ S. cerevisiae Tokay } & $\mathrm{NaCl}$ & $8.59 \pm 0.34$ & $463 \pm 46.08$ \\
\cline { 2 - 4 } & saponin & $6.91 \pm 0.36$ & $883 \pm 66.73$ \\
\hline \multirow{2}{*}{ S. cerevisiae TT } & $\mathrm{NaCl}$ & $7.87 \pm 0.22$ & $2102.33 \pm 76.78$ \\
\cline { 2 - 4 } & saponin & $3.38 \pm 0.16$ & $1654 \pm 66.13$ \\
\hline
\end{tabular}

The characteristics of individual yeast strains were comparable, regardless of the inductor used. The activity of glucanases in the case of Saccharomyces spp. resulted in increased content of mannose and glucose equal to $11 \mathrm{mg} / \mathrm{mL}$ and within the range 33 to $85 \mathrm{mg} / \mathrm{mL}$ after action of saponin, respectively. In the case of $\mathrm{NaCl}$ treatment, carbohydrate content was lower ( 9.5 and 29 to $56 \mathrm{mg} / \mathrm{mL}$, respectively). It is worth noting that the mannose and glucose content in Metschnikowia spp. was close to zero if no glucanase activity detected. The protein and FAN contents were also both strain- and inducerdependent. More protein was determined in the lysates obtained with $\mathrm{NaCl}$. These results differ from those of earlier studies for the strain Saccharomyces cerevisiae with Ethanol Red® (Lesaffre) by Berlowska et al. (2015). 
Table 3 shows the amino-acid profiles of two yeast strains, $M$. andauensis D2 and S. cerevisiae $\mathrm{TT}$, obtained by $\mathrm{NaCl}$ or saponin treatment. These yeasts were chosen due to the fact that their lysates contained the highest amounts of proteins and FAN (Table 2).

Table 3. Amino Acid Profiles of Yeast Lysates

\begin{tabular}{|c|c|c|c|c|}
\hline \multirow{2}{*}{$\begin{array}{c}\text { Amino acid } \\
\text { (pmol) }\end{array}$} & \multicolumn{2}{|c|}{ D2 } & \multicolumn{2}{c|}{ TT } \\
\cline { 2 - 5 } & $\mathbf{N a C l}$ & Saponin & $\mathbf{N a C l}$ & saponin \\
\hline Asp & $17.15 \pm 0.16$ & $141.11 \pm 9.46$ & $562.98 \pm 7.12$ & $587.12 \pm 37.96$ \\
Glu & $591.60 \pm 14.11$ & $1624.44 \pm 17.02$ & $1034.59 \pm 45.23$ & $1077.04 \pm 49.19$ \\
HPro & $\mathbf{3 . 0 3} \pm \mathbf{0 . 0 6}$ & $\mathbf{4 . 2 6} \pm \mathbf{0 . 1 1}$ & $\mathbf{0 . 7 2} \pm \mathbf{0 . 0 7}$ & $\mathbf{0 . 8 0} \pm \mathbf{0 . 0 5}$ \\
Ser & $218.14 \pm 7.10$ & $49.01 \pm 6.12$ & $778.93 \pm 65.11$ & $797.93 \pm 43.09$ \\
Gly & $450.34 \pm 12.16$ & $410.37 \pm 4.11$ & $1019.29 \pm 89.56$ & $1057.36 \pm 76.75$ \\
His & $495.31 \pm 14.19$ & $391.87 \pm 7.36$ & $477.46 \pm 32.06$ & $497.64 \pm 24.16$ \\
Arg & $770.45 \pm 30.17$ & $4.45 \pm 0.16$ & $760.04 \pm 27.78$ & $731.86 \pm 40.11$ \\
Thr & $238.92 \pm 22.10$ & $84.58 \pm 7.13$ & $1160.43 \pm 58.13$ & $1060.62 \pm 65.06$ \\
Ala & $1496.34 \pm 87.12$ & $1075.78 \pm 16.17$ & $2301.05 \pm 79.37$ & $2298.63 \pm 97.19$ \\
Pro & $\mathbf{1 1 3 5 . 2 7} \pm \mathbf{3 8 . 1 3}$ & $\mathbf{1 3 3 3 . 2 8} \pm 64.16$ & $\mathbf{9 6 7 . 0 4} \pm \mathbf{8 7 . 7 6}$ & $\mathbf{1 0 1 1 . 1 7} \pm \mathbf{5 5 . 1 6}$ \\
Tyr & $202.31 \pm 9.16$ & $88.71 \pm 3,11$ & $341.47 \pm 19.10$ & $340.77 \pm 10.16$ \\
Val & $1067.71 \pm 26.14$ & $752,30 \pm 12.06$ & $2336,99 \pm 97,72$ & $2432.03 \pm 123.09$ \\
Met & $79.81 \pm 8.16$ & $65.97 \pm 7.10$ & $535.86 \pm 13.14$ & $557.21 \pm 54.14$ \\
Cys-Cys & $22.35 \pm 7.12$ & $36.40 \pm 2.16$ & $27.22 \pm 4.19$ & $28.03 \pm 2.46$ \\
Ile & $676.32 \pm 32.16$ & $413.97 \pm 10.18$ & $1975.91 \pm 43.17$ & $2054.94 \pm 100.12$ \\
Leu & $1067.71 \pm 53.17$ & $708.52 \pm 14.13$ & $2719.50 \pm 99.03$ & $2831.51 \pm 45.16$ \\
Phe & $265.49 \pm 11.11$ & $116.84 \pm 9.06$ & $1294.31 \pm 42.07$ & $1310.80 \pm 63.34$ \\
Lys & $736.02 \pm 15.12$ & $183.87 \pm 7.89$ & $477.27 \pm 26.16$ & $497.51 \pm 31.17$ \\
\hline Sum & 9534,27 & 7485,73 & $\mathbf{1 8 7 7 1 , 0 6}$ & $\mathbf{1 9 1 7 2 , 9 7}$ \\
\hline
\end{tabular}

The autolysates of the yeast Metschnikowia sp. show a different chemical profile for free amino acids. The pool of amino acids in the D2 autolysate was two times lower than that of the TT autolysate. In general, the obtained amino acid profiles of Metschnikowia strain were dominated by alanine, proline, valine, and leucine, while in Saccharomyces autolizate, mainly glutamic acid, glycine, threonine, alanine, valine, isoleucine, leucine and phenylalanine were detected. The both yeast lysates obtained were found to be good sources of free essential amino acids. These valuable compounds accounted for $43 \%$ (for $M$. andauensis), and $56 \%$ (for $S$. cerevisiae) of the respective amino acid pools. However, it is interesting that the content of hydroxyproline HPro in the Metschnikowia sp. preparation was 4 to 5 times higher. Hydroxyproline-rich glycoproteins are key protein constituents of the cell wall (Lee et al. 2007). Recent discoveries have revealed that hydroxyproline and proline play important roles in growth and differentiation across the life cycle. These amino acids are important components of cell wall proteins, which play pivotal roles in cell wall signal transduction cascades, cell development, and stress tolerance (Nasuno et al. 2013; Kavi Kishor et al. 2015). Proline and hydroxyproline not only participate in protein synthesis, but also regulate several important functions including replicative lifespan, osmotic adjustment, and protection during stress conditions (Mukai et al. 2019). Metschnikowia spp. are known for their unique adaptive abilities, and in this respect differ significantly from the more sensitive Saccharomyces spp. (Pawlikowska and Kregiel 2017). Deficient proline biosynthesis leads to abnormal cells and cell wall defects, indicating its important role in the formation of structural proteins (Kishor et al. 2015). In Metschnikowia sp., proline increases the production of a pigment, 
pulcherrimin, which improves tolerance to oxidative stress and biocontrol (Liu et al. 2019; Pawlikowska et al. 2019b). It is worth noting that Metschnikowia strains show high antifungal activity, which means they compete with other fungal microflora (Sipiczki 2006; Pawlikowska et al. 2019a). These data may explain the increased hydroxyproline content of Metschnikowia yeast lysates.

Lysates of Metschnikowia spp. with a high hydroxyproline content may find applications in biotechnology. Proline regulates several important functions in higher plants, such as osmotic adjustment and protection under stress conditions (Kishor et al. 2015). Hydroxyproline and proline act as biostimulants, penetrating rapidly into plant tissues. The mode of action of complex preparations is varied and may include the activation of nitrogen metabolism, phosphorus release from soils, generic stimulation of soil microbial activity or the stimulation of root growth, and enhanced plant establishment (Yakhin et al. 2017). Proline and hydroxyproline can be considered as functional amino acids for mammalian, avian, and aquatic species. Research on young pigs has shown that proline supplementation improved the daily growth rate and feed efficiency. Dietary supplementation of a plant protein-based diet with hydroxyproline additionally enhanced weight gain in salmon (Wu et al. 2011). Hydroxyproline is the main component of collagen in mammals (Gordon and Hahn 2010), and both proline and hydroxyproline are critical to the mechanical strength of this extracellular matrix molecule. Therefore, Metschnikowia spp. lysates could also be used in the cosmetic industry as regenerating, revitalizing, smoothing, and moisturizing agents (Asserin et al. 2015).

\section{CONCLUSIONS}

1. Metschnikowia lysates show different chemical profiles in comparison to Saccharomyces spp. The pool of individual amino acids was generally lower. However, the content of hydroxyproline was 4 to 5 times higher.

2. Metschnikowia sp. lysates can be used in the form of mono-autolysates or can enrich autolysates of the classical yeast $S$. cerevisiae deficient in hydroxyproline.

3. Lysates of Metschnikowia sp. with high hydroxyproline contents may find application in biotechnology, particularly in the agriculture and cosmetics industries.

\section{ACKNOWLEDGMENTS}

The authors are grateful for the support of NCBiR BIOSTRATEG II: 501/13-1710-6369 "Processing of waste biomass in associated biological - chemical processes".

\section{REFERENCES CITED}

Arino, J. (2002). "Novel protein phosphatases in yeast," Eur. J. Biochem. 269(4), 10721077. DOI: 10.1046/j.0014-2956.2002.02753.x

Asserin, J., Lati, E., Shioya, T., and Prawitt, J. (2015). "The effect of oral collagen peptide supplementation on skin moisture and the dermal collagen network: Evidence 
from an ex vivo model and randomized, placebo-controlled clinical trials," J. Cosmet. Dermatol. 14(4), 291-301. DOI: 10.1111/jocd.12174

Bartolo-Aguilar, Y., Dendooven, L., Chávez-Cabrera, C., Flores-Cotera, L. B., HidalgoLara, M. E., Villa Tanaca, L., and Marsch, R. (2017). "Autolysis of Pichia pastoris induced by cold," AMB Expr. 7:95. DOI:10.1186/s13568-017-0397-y

Berlowska, J., Dudkiewicz, M., Kregiel, D., Czyzowska, A., and Witonska, I. (2015). "Cell lysis induced by membrane-damaging detergent saponins from Quillaja saponaria," Enzyme Microb. Tech. 75-76 (7-8), 44-48. DOI: /10.1016/j.enzmictec.2015.04.007

Berlowska, J., Dudkiewicz-Kołodziejska, M., Pawlikowska, E., Pielech-Przybylska, K., Balcerek, M., Czyzowska, A., and Kregiel, D. (2017). 'Utilization of postfermentation yeasts for yeast extract production by autolysis: The effect of yeast strain and saponin from Quillaja saponaria," J. Inst. Brew. 123(3), 396-401. DOI: 10.1002/jib.438

Bertolo, A. P., Biz, A. P., Kempka, A. P., Rigo, E., and Cavalheiro, D. (2019). "Yeast (Saccharomyces cerevisiae): Evaluation of cellular disruption processes, chemical composition, functional properties and digestibility," J. Food Sci. Technol. 56(8), 3697-3706. DOI: 10.1007/s13197-019-03833-3

Bourdichon, F., Casaregola, S., Farrokh, C., Frisvad, J. C., Gerds, M. L., Hammes, W. P., Harnett, J., Huys, G., Laulund, S., Ouwehand, A., Powell, I. B., Prajapati, J. B., Seto, Y., Schure, E. T., Van Boven, A., Vankerckhoven, V., Zgoda, A., Tuijtelaars, S., and Hansen, E. B. (2012). "Food fermentations: Microorganisms with technological beneficial use," Int. J. Food Microbiol. 154(3), 87-97. DOI: 10.1016/j.ijfoodmicro.2011.12.030

Comuzzo, P., Calligaris, S., Iacumin, L., Ginaldi, F., Voce, S., and Zironi, R. (2017). "Application of multi-pass high pressure homogenization under variable temperature regimes to induce autolysis of wine yeasts," Food Chem. 224, 105-113. DOI: 10.1016/j.foodchem.2016.12.038

Gordon, M. K., and Hahn, R. A. (2010). “Collagens,” Cell Tissue Res. 339(1), 247-257. DOI: $10.1007 / \mathrm{s} 00441-009-0844-4$

Jacob, F. F., Hutzler, M., and Methner, F. J. (2019). "Comparison of various industrially applicable disruption methods to produce yeast extract using spent yeast from topfermenting beer production: influence on amino acid and protein content," Eur. Food Res. Technol. 245(1), 95-109. DOI: 10.1007/s00217-018-3143-Z

Kavi Kishor, P. B., Kumari, H. P., Sunita, M. S., and Sreenivasulu, N. (2015). "Role of proline in cell wall synthesis and plant development and its implications in plant ontogeny," Front. Plant Sci. 6, 544. DOI: 10.3389/fpls.2015.00544

Liu, Y., Yi, L., Ruan, C., Yao, S., Deng, L., and Zeng, K. (2019). "Proline increases pigment production to improve oxidative stress tolerance and biocontrol ability of Metschnikowia citriensis," Front. Microbiol. 10, 1273. DOI: 10.3389/fmicb.2019.01273

Lukondeh, T., Ashbolt, N. J., and Rogers, P. L. (2003). "Evaluation of Kluyveromyces marxianus as a source of yeast autolysates," J. Ind. Microbiol. Biotechnol. 30(1), 5256. DOI: 10.1007/s10295-002-0008-y

Mas, A., Guillamón, J. M., and Beltran, G. (2016). "Editorial: Non-conventional yeast in the wine industry," Front. Microbiol. 7, 1494. DOI: 10.3389/fmicb.2016.01494

Mukai, Y., Kamei, Y., Liu, X., Jiang, S., Sugimoto, Y., Mat Nanyan, N. S. B, Watanabe, D., and Takagi, H. (2019). "Proline metabolism regulates replicative lifespan in the 
yeast Saccharomyces cerevisiae," Microb. Cell 6(10), 482-490. DOI:

10.15698/mic2019.10.694

Nasuno, R., Hirano, Y., Itoh, T., Hakoshima, T., Hibi, T., and Takagi, H. (2013).

"Structural and functional analysis of the yeast N-acetyltransferase Mpr1 involved in oxidative stress tolerance via proline metabolism," Proc. Natl. Acad. Sci. U S A. 110(29), 11821-11826. DOI: 10.1073/pnas.1300558110

Pawlikowska, E., and Kregiel, D. (2017). "Non-conventional yeast Metschnikowia pulcherrima and its application in biotechnology," Post. Mikrobiol. 56(4), 405-415. http://www.pm.microbiology.pl

Pawlikowska, E., James, S. A., Breierova, E., Antolak, H., and Kregiel, D. (2019a). "Biocontrol capability of local Metschnikowia sp. isolates," Antonie Van Leeuwenhoek, 112(10), 1425-1445. DOI: 10.1007/s10482-019-01272-w

Pawlikowska, E., Domanski, J., Dziugan, P., Berlowska, J., Cieciura-Wloch, W., Smigielski, K., and Kregiel, D. (2019b). "Comparison of three deoxidation agents for ozonated broths used in anaerobic biotechnological processes," Processes 7(2), 65. DOI: $10.3390 /$ pr7020065

Pozo-Dengra, J., Martínez-Rodríguez, S., Martínez-Gómez, A. I., Las Heras-Vázquez, F. J., Rodríguez-Vico, F., and Clemente-Jiménez, J. M. (2006). "Screening of autolytic yeast strains for production of 1-amino acids," Enzyme Microb. Tech. 40(1), 46-50. DOI: 10.1016/j.enzmictec.2005.10.036

Ruiz, J., Belda, I., Beisert, B., Navascués, E., Marquina D., Calderón F., Rauhut D., Santos A., Benito S. (2018). "Analytical impact of Metschnikowia pulcherrima in the volatile profile of Verdejo white wines," Appl. Microbiol. Biotechnol. 102(19), 85018509. DOI: $10.1007 / \mathrm{s} 00253-018-9255-3$

Sipiczki, M. (2006). "Metschnikowia strains isolated from botrytized grapes antagonize fungal and bacterial growth by iron depletion," Appl. Environ. Microbiol. 72(10), 6716-6724. DOI: 10.1128/AEM.01275-06

Varela, C., Barker, A., Tran, T., Borneman, A., and Curtin, C. (2017). "Sensory profile and volatile aroma composition of reduced alcohol Merlot wines fermented with Metschnikowia pulcherrima and Saccharomyces uvarum," Int. J. Food Microbiol. 252(Jul 3), 1-9. DOI: 10.1016/j.ijfoodmicro.2017.04.002

Wu, G., Bazer, F. W., Burghardt, R. C., Johnson, G. A., Kim, S. W., Knabe, D. A., Li, P., Li, X., McKnight, J. R., Satterfield, M. C., and Spencer, T. E. (2011). "Proline and hydroxyproline metabolism: implications for animal and human nutrition," Amino Acids 40(4), 1053-1063. DOI: 10.1007/s00726-010-0715-z

Yakhin, O. I., Lubyanov, A. A., Yakhin, I. A., and Brown, P. H. (2017). "Biostimulants in plant science: A global perspective," Front. Plant Sci. 7, 2049. DOI: 10.3389/fpls.2016.02049

Article submitted: December 12, 2019; Peer review completed: March 3, 2020; Revised version received and accepted: March 10, 2020; Published: March 20, 2020.

DOI: $10.15376 /$ biores. 15.2.3228-3236 